\title{
Ảnh hưởng nhận thức khởi nghiệp đến hành vi khởi nghiệp của sinh viên việt nam: Vai trò ý định mục tiêu và ý định hành động \\ Impact of entrepreneurial perceive on entrepreneurial behaviors of Vietnamese students: Role of goal intention and implementation intention
}

\author{
Nguyễn Quang Thu ${ }^{1 *}$, Trần Thế Hoàng ${ }^{1}$, Hà Kiên Tân ${ }^{1}$ \\ ${ }^{1}$ Trường Đại học Kinh tế Thành phố Hồ Chí Minh, Việt Nam \\ *Tác giả liên hệ, Email: ngthu @ueh.edu.vn
}

\begin{tabular}{|c|c|}
\hline THÔNG TIN & TÓM TÁ́T \\
\hline $\begin{array}{l}\text { DOI:10.46223/HCMCOUJS. } \\
\text { econ.vi.13.2.510.2018 }\end{array}$ & $\begin{array}{l}\text { Nghiên cứu này kiểm định mối quan hệ giữa nhận thức, ý } \\
\text { định và hành vi khởi nghiệp của sinh viên Việt Nam thông qua } \\
\text { việc khảo sát } 368 \text { sinh viên đã từng khởi nghiệp tại các trường }\end{array}$ \\
\hline Ngày nhận: 20/01/2018 & Đại học tại Thành phố Hồ Chí Minh, Bình Dương, Đồng Nai, Bà \\
\hline Ngày nhận lại: 02/02/2018 & Rịa Vũng Tàu. Mục tiêu của nghiên cứu này mở rộng lý thuyết \\
\hline Duyệt đăng: 12/03/2018 & $\begin{array}{l}\text { định hành động. Kết quả nghiên cứu cho thấy yếu tố này đều có } \\
\text { tạc động dương đến hành vi khởi nghiệp. Cuối cùng, nghiên cứu }\end{array}$ \\
\hline Tù khóa: & đã đưa ra kết luận và hàm ý chính sách cho các trường Đại học \\
\hline $\begin{array}{l}\text { hành vi khởi nghiệp, nhận } \\
\text { thức khả thi, nhận thức mong } \\
\text { muốn, ý định hănh động, ý }\end{array}$ & ABSTRACT \\
\hline định mục tiêu & $\begin{array}{l}\text { This study investigated the relationships between perceive, } \\
\text { intention and entrepreneurial behaviors of Vietnamese students. } \\
\text { We surveyed } 368 \text { start-up students from universities of Ho Chi }\end{array}$ \\
\hline $\begin{array}{l}\text { Keywords: } \\
\text { entrepreneurial behavior, } \\
\text { perceived feasibility, } \\
\text { perceived desirability, } \\
\text { implementation intention, } \\
\text { goal intention }\end{array}$ & $\begin{array}{l}\text { Minh City, Binh Duong, Dong Nai, and Ba Ria Vung Tau. The } \\
\text { objective of this study extended the EEM theory by adding the } \\
\text { implementation intention to the model. The results showed that } \\
\text { this factor had a positive effect on entrepreneurial behavior. } \\
\text { Finally, we drew the conclusions and implications for university } \\
\text { policies and the future research. }\end{array}$ \\
\hline
\end{tabular}

\section{Giới thiệu}

Khởi nghiệp là một lĩnh vực luôn được các nhà nghiên cứu quan tâm hàng đầu vì sự phát triển nền kinh tế quốc gia. Việc gia tăng được số lượng các doanh nghiệp trong nền kinh tế luôn là mối bận tâm chính của chính phủ, các nhà hoạch định chính sách và các học giả với hai lý do. Một là, tăng tốc độ tăng trưởng kinh tế; Hai là, giảm thất nghiệp (Carree, Piergiovanni, Santarelli, \& Verheul, 2009), đặc biệt với sinh viên mới ra trường (Fayolle \& Liñán, 2014) tại 
các nước đang phát triển. Lứa tuổi thanh niên từ 18 đến 36 thường mạo hiểm, ít sợ rủi ro, mong muốn làm giàu, nhạy bén với các cơ hội kinh doanh, có ý định khởi nghiệp và thực hiện khởi nghiệp ở mức cao (GEM, 2016) ${ }^{1}$.

Tuy nhiên, nhận thức khởi nghiệp của Sinh viên Việt Nam có một số khác biệt nhất định. Tại nhiều quốc gia phát triển, khởi nghiệp dựa trên nền tảng của sự sáng tạo. Trong khi đó, nhận thức tại Việt Nam lại có phần nghiêng về tạo việc làm, tăng thu nhập và xem như là một lựa chọn nghề nghiệp (GEM, 2016). Mặc dù nhận thức về khởi nghiệp ở độ tuổi 18-36 là khá cao, nhưng ý định khởi nghiệp lại không tương xứng (GEM, 2016). Vậy, nhận thức khởi nghiệp của sinh viên có ảnh hưởng như thế nào đến ý định và cuối cùng đến hành vi khởi nghiệp thực sự của họ?

Nghiên cứu này nhằm kiểm định vai trò của nhận thức khởi nghiệp đến ý định và hành vi khởi nghiệp của sinh viên Việt Nam thông qua việc khảo sát sinh viên năm cuối các trường Đại học tại Thành phố Hồ Chí Minh, Bình Dương, Đồng Nai, Bà Rịa Vũng Tàu, với 3 đóng góp mới:

1) Kiểm định vai trò của yếu tố nhận thức (nhận thức khả thi và nhận thức mong muốn) đến ý định (mục tiêu, hành động) và hành vi khởi nghiệp;

2) Kiểm định tác động của yếu tố ý định mục tiêu đến ý định hành động;

3) Đánh giá mức độ tác động của ý định khởi nghiệp (mục tiêu và hành động) đến hành vi khởi nghiệp.

Các phần tiếp theo của nghiên cứu này gồm: (1) Cơ sở lý thuyết và lược khảo các công trình nghiên cứu liên quan; (2) Phương pháp nghiên cứu; (3) Kết quả và thảo luận; (4) Kết luận và hàm ý chính sách.

\section{Lược khảo lý thuyết về khởi nghiệp}

\subsection{Co' sở lý thuyết}

2.1.1. Mô hình sụ kiện khởi nghiệp (Krueger, Reilly, \& Carsrud, 2000)

Theo mô hình này thì ý định khởi nghiệp có liên quan đến nhiều yếu tố, chẳng hạn như sự mong muốn có nhận thức (nhận thức cơ hội khởi nghiệp hay sự hấp dẫn của cơ hội), khuynh hướng hành động và nhận thức tính khả thi (nhận thức về năng lực của nhà khởi nghiệp). Để dự định có thể được thực hiện nó cần phải có một số tác nhân đẩy (tiêu cực), làm thay đổi cuộc sống con người như: bất mãn công việc hiện tại, li dị, mất việc, ... Hay, một số tác nhân kéo theo (tích cực) như tìm được đối tác tốt hoặc có hỗ trợ tài chính. 1997)

2.1.2. Lý thuyết về các pha hành động (Gollwitzer, 1993; Gollwitzer \& Brandstätter,

Lý thuyết các pha hành động (Theory of action phases - TAP) được Gollwitzer (1993) phát triển trong những năm 90. Có hai loại ý định: ý định mục tiêu (goal intention) và ý định hành động (implementation intention). Ý định mục tiêu đề cập đến "Tôi định thực hiện X", trong khi ý định hành động tương ứng với "Tôi có ý định hành động nhắm mục tiêu $\mathrm{X}$ khi tôi

\footnotetext{
${ }^{1}$ GEM: Global Entrepreneurship Monitor - chỉ số khởi nghiệp toàn cầu
} 
gặp tình huống Y”. Vì vậy, những người thiết lập ý định hành động xác định khi nào, ở đâu và làm thế nào họ có kế hoạch ban hành ý định của họ (Brandstätter, Lengfelder, \& Gollwitzer, 2001) từ đó dẫn đến hành động. Như vậy, ý định mục tiêu sẽ ở dạng tổng quát (là một dạng tín hiệu), còn ý định hành động là ý định chi tiết để cụ thể hóa ý định mục tiêu.

\subsubsection{Lý thuyết thiết lập mục tiêu (Latham \& Locke, 1991)}

Lý thuyết thiết lập mục tiêu (Theory of goal setting motivation) cho rằng có hai yếu tố quyết định nhận thức về hành vi: Giá trị và ý định. Trong khi muc tiêu được định nghĩa là nhũng gì cá nhân ý thức được và cố gắng thực hiện. Còn giá trị là nhũng kết quả mà cá nhân đó mong muốn đạt được. Các mục tiêu cần phải rõ ràng cụ thể (không chấp nhận mơ hồ) nhưng cũng phải đầy thách thức sẽ giúp nâng cao động cơ của cá nhân thực hiện để đạt được nó. Mục tiêu càng chi tiết thì càng làm động cơ thực hiện hành vi càng cao.

\subsection{Lực khảo các công trình nghiên cúu thục nghiệm có liên quan}

Các nghiên cứu thực nghiệm trong và ngoài nước có liên quan đến nghiên cứu này được trình bày tóm tắt ở Bảng 1 .

\section{Bảng 1}

Lược khảo tóm tắt một số công trình nghiên cứu liên quan

\begin{tabular}{|c|c|c|}
\hline Nghiên cứu & Lý thuyết nền & Nhận xét \\
\hline $\begin{array}{l}\text { van Gelderen và cộng sự } \\
\text { (2008); } \\
\text { do Paço, Ferreira, Raposo, } \\
\text { Rodrigues, và Dinis (2011); } \\
\text { Bui và Nguyen (2016) }\end{array}$ & $\begin{array}{l}\text { Lý thuyết dự định hành vi } \\
\text { (TPB) Ajzen (1991); Lý } \\
\text { thuyết sự kiện khởi nghiệp } \\
\text { (Krueger et al., 2000) }\end{array}$ & $\begin{array}{l}\text { Sử dụng các yếu tố có sẵn } \\
\text { trong lý thuyết để kiểm định } \\
\text { cho sinh viên Đại học hoạ́c } \\
\text { trung học đến từ các nước } \\
\text { phát triển (Đức, Pháp, Tây } \\
\text { Ban Nha, Bồ Đào Nha) hoặc } \\
\text { các nước có nền kinh tế đang } \\
\text { phát triển (Romania, Ukraine } \\
\text { Malaysia, Trung Quốc, } \\
\text { Tunisia, Ethiopia, } \\
\text { Afghanistan, Peru, Việt } \\
\text { Nam) }\end{array}$ \\
\hline $\begin{array}{l}\text { Hmieleski và Corbett }(2006) ; \\
\text { Hayton và Cholakova } \\
(2012) ;\end{array}$ & $\begin{array}{l}\text { Lý thuyết dự định hành vi } \\
\text { (TPB) Ajzen (1991); }\end{array}$ & $\begin{array}{l}\text { Bổ sung yếu tố yếu tố sự thích } \\
\text { ứng (proclivity for } \\
\text { improvisation) vào mô hình } \\
\text { gốc }\end{array}$ \\
\hline $\begin{array}{l}\text { De Clercq, Dimov, và } \\
\text { Thongpapanl (2013) }\end{array}$ & $\begin{array}{l}\text { Lý thuyết dự định hành vi } \\
\text { (TPB) Ajzen (1991); Lý } \\
\text { thuyết sự kiện khởi nghiệp } \\
\text { (Krueger et al., 2000) }\end{array}$ & $\begin{array}{l}\text { Nghiên cứu mối quan hệ giữa } \\
\text { khả năng nhận thức và sự hấp } \\
\text { dẫn đối với ý định được điều } \\
\text { tiết bởi định hướng học tập và } \\
\text { niềm đam mê làm việc }\end{array}$ \\
\hline $\begin{array}{l}\text { Fitzsimmons và Douglas } \\
\text { (2011); }\end{array}$ & $\begin{array}{l}\text { Lý thuyết dự định hành vi } \\
\text { (TPB) Ajzen (1991); Lý } \\
\text { thuyết sự kiện khởi nghiệp } \\
\text { (Krueger et al., 2000) }\end{array}$ & $\begin{array}{l}\text { Nghiên cứu sự tương tác giữa } \\
\text { nhận thức cơ hội khởi nghiệp } \\
\text { và tính khả thi }\end{array}$ \\
\hline
\end{tabular}




\begin{tabular}{|c|c|c|}
\hline Nghiên cứu & Lý thuyết nền & Nhận xét \\
\hline Nabi và Liñán (2013) & $\begin{array}{l}\text { Lý thuyết dự định hành vi } \\
\text { (TPB) Ajzen (1991) }\end{array}$ & $\begin{array}{l}\text { Nghiên cứu ảnh hưởng của cá } \\
\text { tính, trạng thái tâm lý và nhân } \\
\text { khẩu học đến ý định khởi } \\
\text { nghiệp }\end{array}$ \\
\hline $\begin{array}{l}\text { Bhandari (2012); } \\
\text { (Hadjimanolis \& } \\
\text { Poutziouris, 2011) }\end{array}$ & $\begin{array}{l}\text { Lý thuyết dự định hành vi } \\
\text { (TPB) Ajzen (1991) }\end{array}$ & $\begin{array}{l}\text { Mối cứu mối quan hệ giữa } \\
\text { nền tảng gia đình và ý định } \\
\text { khởi nghiệp }\end{array}$ \\
\hline $\begin{array}{l}\text { Guerrero, Josep, và Urbano } \\
\text { (2008); Bui và Nguyen } \\
\text { (2016) }\end{array}$ & $\begin{array}{l}\text { Lý thuyết dự định hành vi } \\
\text { (TPB) Ajzen (1991) }\end{array}$ & $\begin{array}{l}\text { Nghiên cứu mối quan hệ giữa } \\
\text { nền tảng giáo dục và ý định } \\
\text { khởi nghiệp }\end{array}$ \\
\hline Gird và Bagraim (2008) & $\begin{array}{l}\text { Lý thuyết dự định hành vi } \\
\text { (TPB) Ajzen (1991) }\end{array}$ & $\begin{array}{l}\text { Nghiên cứu mối quan hệ giữa } \\
\text { yếu tố kinh nghiệm và ý định } \\
\text { khởi nghiệp }\end{array}$ \\
\hline $\begin{array}{l}\text { Walker, Jeger, và Kopecki } \\
\text { (2013) }\end{array}$ & $\begin{array}{l}\text { Lý thuyết dự định hành vi } \\
\text { (TPB) Ajzen (1991); Lý } \\
\text { thuyết sự kiện khởi nghiệp } \\
\text { (Krueger et al., 2000) }\end{array}$ & $\begin{array}{l}\text { Các yếu tố môi trường, văn } \\
\text { hóa, thể chế và các tổ chức hô̂ } \\
\text { trợ khởi nghiệp ảnh hưởng } \\
\text { đến ý định khởi nghiệp }\end{array}$ \\
\hline
\end{tabular}

Nguồn: Tổng hợp của nhóm nghiên cứu

Như vậy, các nghiên cứu trên chỉ dừng ở ý định khởi nghiệp. Hướng nghiên cứu từ ý định đến hành vi khởi nghiệp cũng đã được đề cập nhưng chưa có nghiên cứu thực nghiệm nào. Hơn nữa, nhận thức khởi nghiệp của Sinh viên Việt Nam (là quốc gia có nền kinh tế chuyển đổi) có một số khác biệt nhất định so với các nước đang phát triển. Vì vậy, kiểm định mối quan hệ giữa nhận thức đến ý định và hành vi khởi nghiệp được đặt ra để nghiên cứu.

\subsection{Các khái niệm trong mô hình nghiên cúu và các giả thuyết}

\section{Hành vi khởi nghiệp}

Theo Schumpeter (1934), khởi nghiệp là tạo ra các kết hợp mới. Với Cole (1968), là hoạt động có mục đích để khởi đầu, duy trì và phát triển một hoạt động kinh doanh nhằm thu được lợi ích về tài chính hay các lợi ích khác trong một thế giới kinh tế hay kinh doanh mà thế giới đó sẽ tạo ra một sự tự do cho chính người thực hiện hoạt động này. Theo Shapero và Sokol (1982) thì tinh thần khởi nghiệp là quá trình mà cá nhân sẵn sàng tiên phong trong việc nắm bắt các cơ hội kinh doanh hấp dẫn và khả thi mà họ nhận biết được. Trong khi đó ý định khởi nghiệp là bước đầu tiên trong quá trình khám phá, sáng tạo, khai thác cơ hội để khởi nghiệp và thành lập doanh nghiệp mới (Gartner, Shaver, Gatewood, \& Katz, 1994).

\section{Ý định khởi nghiệp}

Các định nghĩa về ý định khởi nghiệp được tổng hợp và trình bày trong Bảng 2. 


\section{Bảng 2}

Định nghĩa về ý định khởi nghiệp

\begin{tabular}{ll}
\hline \multicolumn{1}{c}{ Tác giả } & \multicolumn{1}{c}{ Định nghĩa } \\
\hline Bird (1988) & $\begin{array}{l}\text { Ý định khởi nghiệp là một trạng thái của tâm trí nhấn } \\
\text { mạnh đến sự quan tâm cá nhân và kinh nghiệm để thực } \\
\text { hiện việc tạo ra doanh nghiệp mới. }\end{array}$ \\
\hline Tubbs và Ekeberg (1991) & $\begin{array}{l}\text { Ý định khời nghiệp là một đại diện các hành động có kế } \\
\text { hoạch để thực hiện một hành vi kinh doanh. }\end{array}$ \\
\hline Shane và Venkataraman (2000) & $\begin{array}{l}\text { Ý định khởi nghiệp là quá trình nhận dạng, đánh giá, và } \\
\text { khai thác cơ hội kinh doanh }\end{array}$ \\
\hline Reynolds và cộng sự (2005) & $\begin{array}{l}\text { Ý định khởi nghiệp là các gắn kết cá nhân của các doanh } \\
\text { nhân tiềm năng để bắt đầu khởi nghiệp }\end{array}$ \\
\hline Souitaris, Zerbinati, và Al- & $\begin{array}{l}\text { Ý định khởi nghiệp là sự liên quan về ý định của một cá } \\
\text { nhân để bắt đầu một doanh nghiệp }\end{array}$ \\
\hline Laham (2007) &
\end{tabular}

Nguồn: Tổng hợp của nhóm nghiên cứu

Trong nghiên cứu này, nhóm tác giả sẽ sử dụng định nghĩa của Shane và Venkataraman (2000).

\section{Ý định mục tiêu}

Theo Gollwitzer (1993), ý định mục tiêu chính là ý định trong lý thuyết dự định hành vi (TPB) của Azjen hay EEM, với Fayolle và Liñán (2014) ý định mục tiêu là một dạng cam kết và là một bước đi cần thiết trước khi thực hiện ý định và hành vi. Trong lý thuyết thiết lập mục tiêu thì đó là mục tiêu đầy thách thức vì đầy thách thức mới khiến cá nhân quyết tâm đạt được.

\section{Ý định hành động}

Theo Sheeran và Silverman (2003) phân biệt giữa "giai đoạn tạo động lực mà người đó quyết định hành động và một giai đoạn hoạch định (volitional) mà người đó hoạch định làm thế nào để quyết định trở thành hiện thực". Giai đoạn động lực là giai đoạn liên quan đến quá trình nhận dạng cơ hội khởi nghiệp và khả năng khởi nghiệp trong các mô hình ý định. Giai đoạn hoạch định đề cập đến ý định hành động và không được giải quyết trong các mô hình ý định. Tuy nhiên nó vẫn là một phần của quá trình thực hiện hành vi.

\section{Nhận thức khởi nghiệp}

Một cơ hội kinh doanh thường được định nghĩa như là một "tình huống tương lai được coi là hấp dẫn và khả thi" (Stevenson \& Jarillo, 2007). Tính mong muốn đề cập đến giá trị nhận thức hoặc sự hấp dẫn của cơ hội (ví dụ: cơ hội có tiềm năng lợi nhuận cao được đánh giá là rất mong muốn). Tính khả thi đề cập đến khả năng thực thi hay khó khăn của cơ hội (ví dụ: cơ hội nằm trong một thị trường cạnh tranh cao là khả thi hơn là một cơ hội nằm trong một thị trường chỉ với một vài đối thủ cạnh tranh yếu).

\section{Mối quan hệ giữa ý định mục tiêu và hành vi khởi nghiệp}

Lý thuyết về sự kiện khởi nghiệp (EEM) của (Shapero \& Sokol, 1982), lý thuyết hành vi có kế hoạch (TPB) của (Ajzen, 1991) cho rằng ý định là chỉ báo quan trọng dự báo hành vi 
của con người. Theo Randall và Wolff (1994), mối quan hệ giữa ý định và hành vi không thay đổi theo thời gian. Trong nghiên cứu tổng kết về định lượng 98 nghiên cứu trước đó của (Schlaegel \& Koenig, 2014) khẳng định vẫn có tương quan cao cùng chiều giữa ý định và hành vi trong bối cảnh khởi nghiệp. Từ những phân tích nêu trên, giả thuyết H1a được đưa ra như sau:

\section{Hla: Ý định muc tiêu tác động cùng chiều đến hành vi khởi nghiệp}

\section{Mối quan hệ giữa ý định hành động và hành vi khởi nghiệp}

Ý định hành động hiệu quả trong việc dẫn dắt hành động, đặc biệt khi các mục tiêu có thể đạt được thông qua các hành động, hoặc đối với mô hình hành vi tương đối phức tạp như khởi nghiệp. Sheeran và Silverman (2003) xác nhận ý định hành động làm tăng khả năng hành động và hiệu quả của nó cũng không giảm theo thời gian. Các ý định hành động cũng có thể giúp giải quyết một cách hiệu quả sự lo lắng và những cảm xúc tiêu cực (Sheeran, Aubrey, \& Kellett, 2007). Như vậy, căn cứ vào những phân tích trên, giả thuyết $\mathrm{H} 1 \mathrm{~b}$ được phát biểu như sau:

H1b: Ý định hành động sẽ có tác động cùng chiều đến hành vi khởi nghiệp

\section{Mối quan hệ giữa ý định mục tiêu và ý định hành động}

Ý định mục tiêu được theo đuổi sẽ thành công hơn khi kết hợp với các ý định hành động (Gollwitzer \& Brandstätter, 1997). Vì nó tạo ra một liên kết chặt chẽ giữa các tín hiệu và các hành vi trong trí nhớ và mục đích thực hiện sẽ làm tăng khả năng tiếp cận trong tư duy vì thế gây ra hành động (Gollwitzer, 1993). Cho nên, các ý định hành động tạo thuận lợi cho việc bắt đầu các hành vi dự định (Gollwitzer \& Sheeran, 2006). Trên cơ sở lý thuyết của Gollwitzer (1993), giả thuyết H2 được phát biểu như sau:

\section{H2: Ý định mục tiêu sẽ có tác động cùng chiều đến ý định hành động khởi nghiệp}

\section{Mối quan hệ giữa nhận thức khởi nghiệp và ý định mục tiêu}

Các nghiên cứu gần đây cho rằng mong muốn và khả thi là đặc điểm của các cơ hội kinh doanh cụ thể (Haynie, Shepherd, \& McMullen, 2009). Kết quả này cho thấy ảnh hưởng trực tiếp của tính mong đợi và khả thi của việc đánh giá cơ hội (Haynie et al., 2009; Mitchell Shepherd, \& Sharfman, 2011). Hơn nữa tính mong muốn và tính khả thi cao đều có ảnh hưởng tích cực đến việc đánh giá cơ hội. Lý thuyết tiềm năng khởi nghiệp của (Krueger \& Brazeal, 1994) cho rằng một cá nhân ham muốn khởi nghiệp, cảm nhận về tính khả thi khởi nghiệp thì sẽ có tiềm năng khởi nghiệp, nếu có tác động của các nhân tố kéo hoặc đẩy thì tiềm năng khởi nghiệp sẽ thúc đẩy cá nhân hình thành ý định khởi nghiệp. Từ những phân tích nêu trên giả thuyết $\mathrm{H} 3 \mathrm{a}$ và $\mathrm{H} 4 \mathrm{a}$ được phát biểu như sau:

H3a: Nhận thức khả thi sẽ tác động cùng chiều đến ý định mục tiêu khởi nghiệp

H4a: Nhận thức mong muốn sẽ tác động cùng chiều đến ý định muc tiêu khởi nghiệp

\section{Mối quan hệ giữa nhận thức khởi nghiệp và ý định hành động}

Gollwitzer (1993) cho rằng giai đoạn nhận thức chính là giai đoạn tạo động lực để khởi nghiệp. Một cá nhân khi có động lực sẽ hình thành ý định mục tiêu hoặc là ý định hành động. 
Sức mạnh của ý định hành động trong việc nối kết giữa nhận thức, ý định mục tiêu và hành vi lần đầu tiên được Gollwitzer kiểm tra và nhiều nghiên cứu thực nghiệm khác. Nghiên cứu của (Brännback, Carsrud, Elfving, Kickul, \& Krueger, 2006), ý định khởi nghiệp chủ yếu phụ thuộc vào nhận thức cơ hội và nhận thức khả năng khởi nghiệp. Trong nghiên cứu của (Schlaegel \& Koenig, 2014), kết hợp giữa hai mô hình TPB (Ajzen, 1991) và EEM (Krueger et al., 2000). Vì thế, giả thuyết $\mathrm{H} 3 \mathrm{~b}, \mathrm{H} 4 \mathrm{~b}$ được phát biểu như sau:

H3b: Nhận thức khả thi sẽ tác động cùng chiều đến ý định hành động khởi nghiệp H4b: Nhận thức mong muốn sẽ tác động cùng chiều đến ý định hành động khởi nghiệp

Tổng hợp các giả thuyết trên, mô hình nghiên cứu đề xuất được trình bày như Hình 1 :

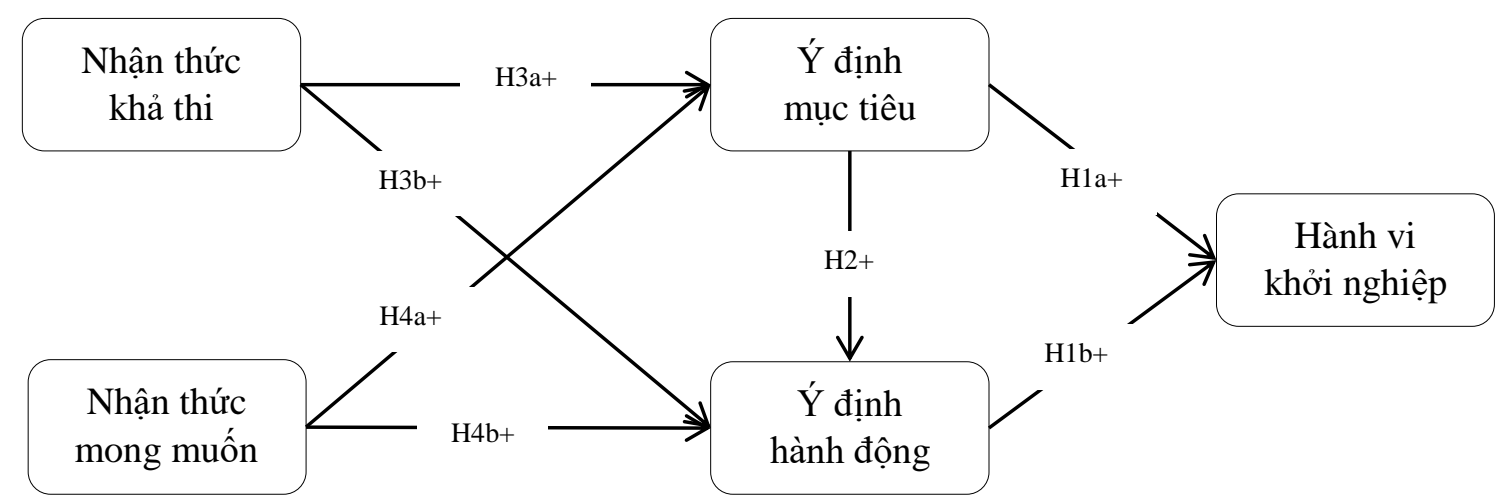

Hình 1. Mô hình nghiên cứu đề xuất

\section{Dữ liệu và phương pháp nghiên cứu}

\subsection{Dũ liệu nghiên cúu}

Nghiên cứu sử dụng nguồn dữ liệu điều tra bằng phỏng vấn trực tiếp thông qua bảng câu hỏi chi tiết với thang đo Likert 7 bậc (từ 1: Hoàn toàn không đồng ý đến 7 : hoàn toàn đồng ý).

\section{Mẫu nghiên cưu}

Nghiên cứu này sử dụng kỹ thuật phỏng vấn trực tiếp bằng bảng câu hỏi chi tiết với sinh viên năm cuối tại Thành phố Hồ Chí Minh, Bình Dương, Đồng Nai, Bà Rịa Vũng Tàu. Thời gian phỏng vấn từ tháng 4-7/2017.

Mẫu được chọn theo phương pháp thuận tiện với số lượng theo nguyên tắc 5:1 (Nguyen, 2011). Mô hình có (22 biến quan sát +02 biến kiểm soát) $* 5=120$. Phát ra 1817 phiếu thu về 1432 phiếu. Trong đó có 406 phiếu trả lời đã và đang khởi nghiệp, trong số này có 38 phiếu có số lượng các câu trả lời trống trên $10 \%$ tổng số câu hoặc các câu chọn điểm như nhau vì vậy bị loại. Số còn lại là 368 được đưa vào nghiên cứu chính thức. Theo đó, Đại học Kinh tế - Kỹ thuật Bình Dương (17SV-4,62\%); Đại học Bình Dương (9SV-2,45\%); Đại học Thủ Dầu Một (20SV-5,43\%); Đại học Lạc Hồng (35SV-9,51\%), Đại học Bà Rịa Vũng Tàu (13SV-3,53\%); Đại học Kinh tế Thành phố Hồ Chí Minh (157SV-42,66\%); Đại học Hoa Sen (33SV-8,97\%); Đại học Tài chính - Marketing (30SV-8,15\%); Đại học Mở Thành phố Hồ Chí Minh (54SV- 
14,67\%). Về ngành nghề chỉ khảo sát sinh viên năm cuối quản trị kinh doanh. Về giới tính, nam chiếm $73,8 \%$ với $271 \mathrm{SV}$, trong khi đó nữ chiếm $26,2 \%$ với $97 \mathrm{SV}$.

\section{Thang đo}

Thang đo dựa trên các thang đo lập lại và được điều chỉnh sau nghiên cứu định tính. Tất cả các thang đo trong mô hình nghiên cứu đều là thang đo đa biến. Các thang đo này sử dụng Likert 7 bậc. Mô hình có 5 khái niệm nghiên cứu với 22 biến quan sát được trình bày trong Bảng 3.

\section{Bảng 3}

Thang đo các khái niệm trong mô hình và nguồn gốc các thang đo

\section{Khái niệm nghiên cứu}

$\begin{gathered}\text { Số biến } \\ \text { quan } \\ \text { sát }\end{gathered} \quad$ Nguồn gốc
.

\section{Ý định mục tiêu khởi nghiệp (INT)}

1) Tôi đã sẵn sàng làm bất cứ điều gì để trở thành một doanh nhân; 2) Mục tiêu nghề nghiệp của tôi đang trở thành một doanh nhân; 3) Tôi sẽ cố gắng hết sức để bắt đầu và điều hành công ty của riêng tôi; 4) Tôi quyết tâm tạo công ty

Krueger et al. (2000);

Wang, $\mathrm{Lu}$, và

Millington (2011) trong tương lai

\section{Hành vi khởi nghiệp (BEH)}

1) Xây dựng kế hoạch kinh doanh; 2) Phát triển sản phẩm hoặc dịch vụ; 3) Các nỗ lực tiếp thị theo kế hoạch; 4) Nói chuyện với khách hàng tiềm năng; 5) Thu thập thông tin về đối thủ cạnh tranh

Kautonen, van

Gelderen, và

Tornikoski (2015)

\section{Ý định hành động khởi nghiệp (INT_I)}

1) Bạn có nghĩ về một ý tưởng có thể làm cơ sở để bắt đầu công ty của riêng bạn; 2) Bạn đang phát triển một sản phẩm/dịch vụ; 3) Hiện bạn đang tiết kiệm tiền cho ý định

Gollwitzer và Brandstätter (1997). bắt đầu kinh doanh của mình

\section{Nhận thức mong muốn (DES)}

1) Là một doanh nhân có nhiều lợi thế hơn bất lợi đối với tôi; 2) Một sự nghiệp như một doanh nhân là điều thú vị đối với tôi; 3 ) nếu tôi có cơ hội, vốn và khả năng, tôi sẽ bắt đầu một công ty mới; 4) Là một doanh nhân sẽ cho tôi một sự hài lòng lớn; 5) Trong số các lựa chọn khác nhau, tôi muốn trở thành một doanh nhân

\section{Nhận thức khả thi (FEA)}

1)Tin tưởng vào khả năng tồn tại và tăng trưởng của dự án kinh doanh nếu tôi có ý định thành lập công ty; 2) Tôi cho rằng việc thành lập công ty với tôi là khả thi; 3)Tôi đủ phẩm chất để thành lập công ty; 4) Kiến thức và kinh nghiệm là động lực cho tôi thành lập công ty mới; 5) Mạng lưới các mối quan hệ thuận lợi cho việc thành lập công ty của tôi 


\subsection{Phương pháp nghiên cúu}

Phương pháp nghiên cứu được thực hiện thông qua 02 giai đoạn: (1) Nghiên cứu sơ bộ; và (2) Nghiên cứu chính thức.

Nghiên cúu so bộ định tính: dùng để điều chỉnh các biến quan sát đo lường các khái niệm nghiên cứu thông qua kỹ thuật thảo luận nhóm để các thang đo có thể được hiểu rõ ràng và đồng nhất về yếu tố nhận thức, ý định mục tiêu, ý định hành động, và hành vi khởi nghiệp.

Nghiên cứu so bộ định lương: Thang đo nháp được dùng để phỏng vấn thử với mẫu 110 sinh viên đã và đang khởi nghiệp theo phương pháp lấy mẫu thuận tiện để kiểm định độ tin cậy của thang đo. Sau bước này, thang đo được hoàn chỉnh và sử dụng cho bước nghiên cứu định lượng chính thức. Nghiên cứu sơ bộ định lượng được thực hiện để đánh giá thang đo bằng hệ số tin cậy (Cronbach's Alpha) và phân tích nhân tố khám phá (EFA).

\section{Kết quả nghiên cứu và thảo luận}

\subsection{Kết quả nghiên cúu}

Kết quả phân tích CFA với mô hình tới hạn cho thấy chi-bình phương là 240,109 với 109 bậc tự do, giá trị $\mathrm{p}=0,000$. Nếu điều chỉnh theo bậc tự do có $\mathrm{CMIN} / \mathrm{df}=2,202<3$, đạt yêu cầu độ tương thích. Các chỉ tiêu khác nhau như GFI $=0,928, \mathrm{TLI}=0,935, \mathrm{CFI}=0.948$, RMSEA $=0.057<0,80$, đều đạt yêu cầu. Vì vậy, mô hình tới hạn đạt được độ tương thích với dữ liệu thị trường.

\section{Bảng 4}

Bảng tóm tắt kết quả kiểm định thang đo

\begin{tabular}{lcccc}
\hline & \multirow{2}{*}{$\begin{array}{c}\text { Số } \\
\text { biến } \\
\text { Kuái niệm }\end{array}$} & \multicolumn{2}{c}{ Độ tin cậy } & $\begin{array}{c}\text { Phương sai } \\
\text { trích } \\
\text { (Average }\end{array}$ \\
\cline { 5 - 6 } & sát & $\begin{array}{c}\text { Cronbach's } \\
\text { Alpha }\end{array}$ & $\begin{array}{c}\text { Tổng hộp } \\
\text { (Composite } \\
\text { Reliability) }\end{array}$ & $\begin{array}{c}\text { Variance } \\
\text { Extracted) }\end{array}$ \\
\hline Ý định mục tiêu & 03 & 0,860 & 0,862 & 0,676 \\
\hline Ý định hành động & 03 & 0,804 & 0,808 & 0,584 \\
\hline Hành vi khởi nghiệp & 03 & 0,788 & 0,761 & 0,485 \\
\hline Nhận thức mong muốn & 04 & 0,756 & 0,806 & 0.517 \\
\hline Nhận thức khả thi & 03 & 0,806 & 0,806 & 0,511 \\
\hline
\end{tabular}

Nguồn: Xử lý số liệu điều tra của nhóm nghiên cứu

Kết quả SEM (Hình 2) cho thấy chi-bình phương là 322,014 với 112 bậc tự do, giá trị $\mathrm{p}=0,000$. Nếu điều chỉnh theo bậc tự do có $\mathrm{CMIN} / \mathrm{df}=2,875<3.0$, đạt yêu cầu độ tương thích; $\mathrm{GFI}=0,907, \mathrm{TLI}=0,898, \mathrm{CFI}=0.916, \mathrm{RMSEA}=0.071<0,80$, đều đạt yêu cầu. Hiện tượng Heywood không xuất hiện trong quá trình ước lượng các mô hình CFA, SEM và đa số các sai số chuẩn (standardized residuals) đều nhỏ hơn $|2,28|$. Vì vậy, có thể kết luận, mô hình này thích hợp với dữ liệu thị trường. 
Chi-square=322.014; $\mathrm{df}=112 ; P=.000$

;Chi-square/df=2.875

GFI=.907; TLI=.898; CFI=.916

;RMSEA $=.071$

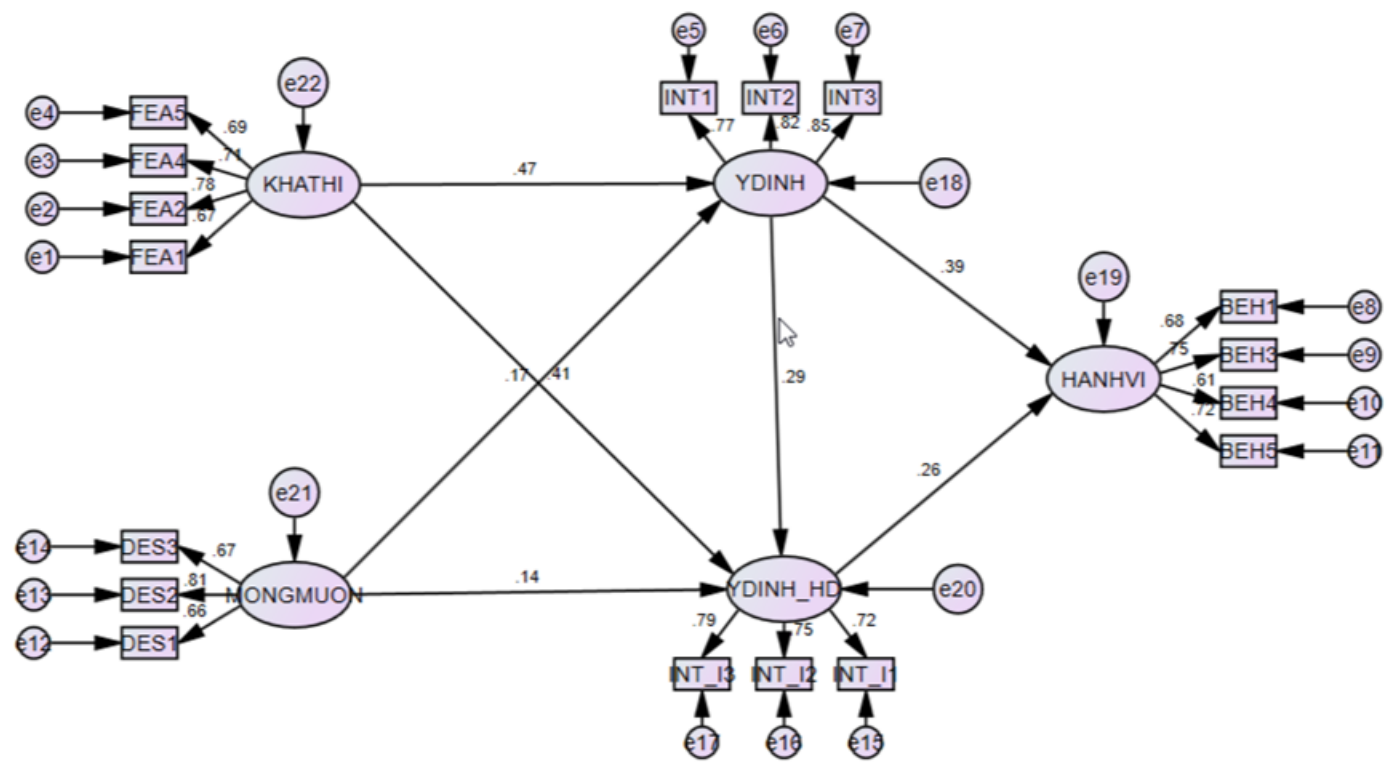

Hình 2. Kết quả SEM cho mô hình lý thuyết (đã chuẩn hóa)

Kết quả ước lượng các tham số chính được trình bày ở Bảng 5 tất cả các mối quan hệ đều có ý nghĩa thống kê (p-value $<5 \%$ ). Như vậy tất cả các giả thuyết đều được chấp nhận.

\section{Bảng 5}

Kết quả kiểm định mối quan hệ giữa các khái niệm nghiên cứu (chuẩn hóa)

\begin{tabular}{lclrrrr}
\hline & \multirow{2}{*}{ Mối quan hệ } & $\begin{array}{c}\text { Ước } \\
\text { lượng }\end{array}$ & S.E. & C.R. & P \\
\hline Hành vi khởi nghiệp & $<---$ & Ý định mục tiêu & 0,351 & 0,066 & 5.306 & $* * *$ \\
\hline Hành vi khởi nghiệp & $<---$ & Ý định hành động & 0,256 & 0,071 & 3.595 & $* * *$ \\
\hline Ý định hành động & $<---$ & Ý định mục tiêu & 0,270 & 0,063 & 4.309 & $* * *$ \\
\hline Ý định mục tiêu & $<---$ & Nhận thức khả thi & 0,570 & 0,082 & 6.963 & $* * *$ \\
\hline Ý định mục tiêu & $<---$ & Nhận thức mong muốn & 0,247 & 0,087 & 2.829 & 0,005 \\
\hline Ý định hành động & $<---$ & Nhận thức khả thi & 0,457 & 0,082 & 5.546 & $* * *$ \\
\hline Ý định hành động & $<---$ & Nhận thức mong muốn & 0,182 & 0,079 & 2.303 & 0,021 \\
\hline
\end{tabular}

Ghi chú: *** mức ý nghĩa $1 \%$

Nguồn: Kết quả từ xử lý số liệu điều tra của nhóm nghiên cứu

Kết quả các tác động trực tiếp, gián tiếp và tổng hợp vào các biến phụ thuộc (hành vi khởi nghiệp, ý định hành động, ý định mục tiêu) được trình bày ở Bảng 6. 


\section{Bảng 6}

Tác động nhận thức khởi nghiệp đến hành vi khởi nghiệp thông qua ý định khởi nghiệp

\begin{tabular}{|c|c|c|c|c|c|}
\hline $\begin{array}{l}\text { Biến phụ } \\
\text { thuộc }\end{array}$ & Loại tác động & $\begin{array}{c}\text { Ý định } \\
\text { mục tiêu }\end{array}$ & $\begin{array}{c}\text { Ý định } \\
\text { hành động }\end{array}$ & $\begin{array}{c}\text { Nhận thức } \\
\text { khả thi }\end{array}$ & $\begin{array}{l}\text { Nhận } \\
\text { thức } \\
\text { mong } \\
\text { muốn }\end{array}$ \\
\hline \multirow{3}{*}{$\begin{array}{l}\text { Hành vi khởi } \\
\text { nghiệp }\end{array}$} & Trực tiếp & 0,394 & 0,264 & 0 & 0 \\
\hline & Gián tiếp & 0,078 & 0 & 0,328 & 0,115 \\
\hline & Tổng hợp & 0,472 & 0,264 & 0,328 & 0,115 \\
\hline \multirow{3}{*}{$\begin{array}{l}\text { Ý định mục } \\
\text { tiêu }\end{array}$} & Trực tiếp & 0 & 0 & 0,467 & 0,168 \\
\hline & Gián tiếp & 0 & 0 & 0 & 0 \\
\hline & Tổng hợp & 0 & 0 & 0,467 & 0,168 \\
\hline \multirow{3}{*}{$\begin{array}{l}\text { Ý định hành } \\
\text { động }\end{array}$} & Trực tiếp & 0,294 & 0 & 0,407 & 0,135 \\
\hline & Gián tiếp & 0 & 0 & 0,137 & 0,049 \\
\hline & Tổng hợp & 0,294 & 0 & 0,544 & 0,184 \\
\hline
\end{tabular}

Nguồn: Kết quả từ xử lý số liệu điều tra của nhóm nghiên cứu

\section{2. Đánh giá kết quả}

Kết quả nghiên cứu có một số ý nghĩa như sau:

Một là, Kiểm chứng được vai trò quan trọng của nhận thức khả thi đến ý định khởi nghiệp (ý định mục tiêu và ý định hành động) của sinh viên Việt Nam.

Hai là, Khẳng định lại vai trò quan trọng của ý định mục tiêu và ý định hành động trong mối quan hệ giữa nhận thức và hành vi khởi nghiệp, trong đó yếu tố trung gian ý định hành động được đưa vào mô hình có ý nghĩa thống kê (mối quan hệ mới chưa được kiểm định tại Việt Nam), góp phần phát triển lý thuyết sự kiện khởi nghiệp (EEM) của Krueger và cộng sự (2000).

Ba là, Yếu tố ý định hành động được xem là yếu tố có hiệu quả đối với việc thúc đẩy hành vi khởi nghiệp. Nó nhấn mạnh từ dạng tâm trí (ý định mục tiêu) chuyển sang ý định hành động nhằm cụ thể hóa các mục tiêu đã định hướng trước đó. Từ đó giúp nhà khởi nghiệp tiềm năng kiên trì với ý định khởi nghiệp.

\section{Kết luận và hàm ý chính sách cho các trường Đại học}

\subsection{Kết luận}

Kết quả nghiên cứu này khác với kết quả nghiên cứu của (Schlaegel \& Koenig, 2014) được kiểm định tại các nước phương Tây) cho rằng yếu tố nhận thức mong muốn (khả năng nhận thức cơ hội của nhà khởi nghiệp) có vai trò rất quan trọng trong việc hình thành ý định hơn là so với yếu tố nhận thức khả thi (nhận thức năng lực của nhà khởi nghiệp). Schlaegel và Koenig (2014) nhận định rằng có thể tại các nước phương Đông nhận thức khả thi sẽ quan trọng hơn so với nhận thức mong muốn và đề nghị kiểm định lại mối quan hệ này. Trong khi đó, kết 
quả của nghiên cứu này (tại Việt Nam) lại cho thấy nhận thức khả thi có tác động rất mạnh đến ý định (cả mục tiêu và hành động) và hành vi khởi nghiệp.

\section{Hạn chế và hướng nghiên cứu tiếp theo}

Nghiên cứu này chỉ thực hiện đối với sinh viên năm cuối ngành quản trị kinh doanh tại 09 trường Đại học các tỉnh/thành: Thành phố Hồ Chí Minh, Bình Dương, Đồng Nai, Bà Rịa Vũng Tàu, do đó khả năng tổng quát hóa chưa cao. Kết quả sẽ tốt hơn nếu được thực hiện tại các trường Đại học như Cần Thơ, Đà Nẵng, Hà Nội.

Nghiên cứu này chọn mẫu thuận tiện do đó cũng có nhược điểm là tính đại diện không cao. Hơn nữa, nghiên cứu từ ý định đến hành vi nếu chỉ khảo sát một lần cũng có hạn chế nhất định, nó cần phải có các dữ liệu một thời điểm và đa thời điểm để phân tích thì sẽ cho kết quả tốt.

Còn nhiều các yếu tố khác tác động đến ý định và đến hành vi khởi nghiệp như các yếu tố văn hóa hoặc động cơ khởi nghiệp, ... đây cũng là một hướng phát triển tiếp theo của nghiên cứu.

\subsection{Hàm ý chính sách và giải pháp}

Kết quả nghiên cứu đã khẳng định yếu tố nhận thức khả thi (năng lực của nhà khởi nghiệp) và ý định hành động có tác động rất lớn đến hành vi khởi nghiệp. Yếu tố này gợi mở cho sinh viên cần trải nghiệm và kiên trì đầu tư cho các ý tưởng khởi nghiệp của mình thông qua các hoạt động đào tạo tại Đại học nhằm tăng cường năng lực khởi nghiệp cho sinh viên. Sau đây là một số hàm ý chính sách và giải pháp:

\section{Đối với sinh viên}

Trước khi khởi nghiệp sinh viên cần phải hình thành động lực thông qua nhận thức tính hấp dẫn của các cơ hội khởi nghiệp, đánh giá năng lực khởi nghiệp từ đó hình thành các ý tưởng, ý định khởi nghiệp cộng với đam mê để thực hiện những ý định khởi nghiệp đó. Những nhà khởi nghiệp thành công ngoài việc có động lực, khát vọng, ý chí thì cần phải kiên trì phát triển các ý tưởng kinh doanh dù phải đối mặt với nhiều trở ngại khó khăn và thất bại. Nghiên cứu này cho thấy, khả năng khởi nghiệp là yếu tố rất quan trọng, trong khi rất nhiều người cố gắng khởi nghiệp còn thiếu những kiến thức, kỹ năng và những năng lực cơ bản. Đây là một trong những lý do mà nhiều nhà khởi nghiệp gặp khó khăn ngay từ đầu hoặc đóng cửa chỉ sau vài tháng khởi nghiệp.

Để có thể khởi nghiệp, sinh viên cần được trang bi rất nhiều nền tảng kiến thức khởi nghiệp có liên quan như kiến thức về thị trường sản phẩm/dịch vụ, bán hàng và marketing, đối thủ cạnh tranh, công nghệ, nguồn nhân lực, luật pháp đặc biệt là tài chính. Nếu sinh viên có ý tưởng, kiên trì, tìm được thị trường cho sản phẩm/dịch vụ của mình nhưng thiếu vốn thì tất cả vẫn chỉ là ý tưởng trên giấy. Vì vậy, nếu không có hoặc ít vốn để khởi nghiệp thì sinh viên có thể huy động vốn bên ngoài nếu muốn khởi nghiệp.

\section{Đối với các trường Đại học}

Các trường Đại học nên tập trung đào tạo phương pháp và kỹ năng để giúp sinh viên phát hiện ra các sản phẩm/dịch vụ đáp ứng nhu cầu thị trường. Hiện nay, sinh viên thường được 
đào tạo theo các quy tắc kinh doanh, các lí thuyết kinh tế, phương pháp tài chính và xây dựng bản kế hoạch kinh doanh khá lý thuyết trong khi khởi nghiệp phải điều hành doanh nghiệp thực.

Sinh viên thường chỉ được dạy làm ra nhiều tiền thay vì được dạy về gia tăng giá trị cho thị trường. Mục đích tối thượng của khởi nghiệp là phá vỡ thị trường bằng việc đem tới giá trị cho nhiều người và đồng thời tạo ra việc làm mới, thị trường mới, và ngành công nghiệp mới.

Các trường Đại học tại Việt Nam chưa có hoạt động tạo môi trường để sinh viên có thể trao đổi các ý tưởng và học hỏi từ người khác, rất khó tìm ra người hỗ trợ giỏi, phần lớn sinh viên đều tự làm việc độc lập. Vì vậy, các trường Đại học nên tạo ra môi trường cho sinh viên thảo luận về các ý tưởng của họ với các doanh nhân thành công, từ đó hiện thực hóa ý tưởng và tiến tới khởi nghiệp. Khởi nghiệp là phải hành động không phải trên giấy. Bên cạnh dạy về tài chính, kinh tế và quản trị thì phải dạy cho sinh viên về tâm lí để có thể đối phó với những thất bại và chấp nhận rủi ro.

\section{Tài liệu tham khảo}

Ajzen, I. (1991). The theory of planned behavior. Organizational Behavior and Human Decision Processes, 50(2), 179-211. doi:10.1016/0749-5978(91)90020-T

Albert, B. (1986). Social foundations of thought and action: A social cognitive theory. New York, NY: Prentice-Hall.

Bhandari, N. C. (2012). Relationship between students' gender, their own employment, their parents' employment, and the students' intention for entrepreneurship. Journal of Entrepreneurship Education, 15, 133-144.

Bird, B. (1988). Implementing entrepreneurial ideas: The case for intention. Academy of Management Review, 13(3), 442-453. doi:10.5465/amr.1988.4306970

Brandstätter, V., Lengfelder, A., \& Gollwitzer, P. M. (2001). Implementation intentions and efficient action initiation. Journal of Personality and Social Psychology, 81(5), 946-960. doi:10.1037/0022-3514.81.5.946

Brännback, M., Carsrud, A., Elfving, J., Kickul, J., \& Krueger, N. F. (2006). Why replicate entrepreneurial intentionality studies? Prospects, perils, and academic reality. Retrieved October 5, 2017, from https://www.researchgate.net/publication/251406483_Why_replicate_entrepreneurial_i ntentionality_studies_Prospects_perils_and_academic_reality

Bui, T. T., \& Nguyen, H. X. (2016). Ý định khởi nghiệp của sinh viên: Nghiên cứu điều tra tại TP.HCM [Student entrepreneurship intentions: An investigation in Ho Chi Minh City]. Paper presente at Hội thảo khoa học: Khởi nghiệp tại Việt Nam: Cơ hội và thách thức trong thời kỳ hội nhập, Đại học Kinh tế Thành phố Hồ Chí Minh, Ho Chi Minh, Vietnam.

Carree, M., Piergiovanni, R., Santarelli, E., \& Verheul, I. (2009). Policies fostering new firm formation and self-employment in Italy. In Public policies for fostering entrepreneurship (pp. 15-34). New York, NY: Springer.

Cole, A. H. (1968). The entreprenuer introductory remarks. The American Economic Review, $58(2), 60-63$. 
De Clercq, D., Dimov, D., \& Thongpapanl, N. (2013). Organizational social capital, formalization, and internal knowledge sharing in entrepreneurial orientation formation. Entrepreneurship Theory and Practice, 37(3), 505-537. doi:10.1111/etap.12021

do Paço, A. M. F., Ferreira, J. J. M., Raposo, M., Rodrigues, R. G., \& Dinis, A. (2011). Behaviours and entrepreneurial intention: Empirical findings about secondary students. Journal of International Entrepreneurship, 9(1), 20-38. doi:10.1007/s10843-010-0071-9

Fayolle, A., \& Liñán, F. (2014). The future of research on entrepreneurial intentions. Journal of Business Research, 67(5), 663-666. doi:10.1016/j.jbusres.2013.11.024

Fitzsimmons, J. R., \& Douglas, E. J. (2011). Interaction between feasibility and desirability in the formation of entrepreneurial intentions. Journal of Business Venturing, 26(4), 431440. doi:10.1016/j.jbusvent.2010.01.001

Gartner, W. B., Shaver, K. G., Gatewood, E., \& Katz, J. A. (1994). Finding the entrepreneur in entrepreneurship. Entrepreneurship Theory and Practice, 18, 5-5.

GEM. (2016). Báo cáo chỉ số khởi nghiệp Việt nam 2015: Hoạt động kinh doanh xã hội [Vietnam startup index 2015 report: Social business activities]. Hanoi, Vietnam: NXB Giao thông Vận tải.

Gird, A., \& Bagraim, J. (2008). The theory of planned behaviour as predictor of entrepreneurial intent amongst final-year university students. South African Journal of Psychology, 38(4), 711-724. doi:10.1177/008124630803800410

Gollwitzer, P. M. (1993). Goal achievement: The role of intentions. European Review of Social Psychology, 4(1), 141-185. doi:10.1080/14792779343000059

Gollwitzer, P. M., \& Brandstätter, V. (1997). Implementation intentions and effective goal pursuit. Journal of Personality and Social Psychology, 73(1), 186-199. doi:10.1037/0022-3514.73.1.186

Gollwitzer, P. M., \& Sheeran, P. (2006). Implementation intentions and goal achievement: A meta analysis of effects and processes. Advances in Experimental Social Psychology, 38, 69-119. doi:10.1016/S0065-2601(06)38002-1

Guerrero, M., Josep, R., \& Urbano, D. (2008). The impact of desirability and feasibility on entrepreneurial intentions: A structural equation model. International Entrepreneurship and Management Journal, 4(1), 35-50. doi:10.1007/s11365-006-0032-x

Hadjimanolis, A., \& Poutziouris, P. (2011). Family business background, perceptions of barriers, and entrepreneurial intentions in Cyprus. International Journal of Entrepreneurial Venturing, 3(2), 168-182. doi:10.1504/IJEV.2011.039339

Haynie, J. M., Shepherd, D. A., \& McMullen, J. S. (2009). An opportunity for me? The role of resources in opportunity evaluation decisions. Journal of Management Studies, 46(3), 337-361.

Hayton, J. C., \& Cholakova, M. (2012). The role of affect in the creation and intentional pursuit of entrepreneurial ideas. Entrepreneurship Theory and Practice, 36(1), 41-68. doi:10.1111/j.1540-6520.2011.00458.x 
Hmieleski, K. M., \& Corbett, A. C. (2006). Proclivity for improvisation as a predictor of entrepreneurial intentions. Journal of Small Business Management, 44, 45-63. doi:10.1111/j.1540-627X.2006.00153.x

Kautonen, T., van Gelderen, M., \& Tornikoski, E. T. (2015). Predicting entrepreneurial behaviour: A test of the theory of planned behaviour. Applied Economics, 45(6), 697707. doi:10.1080/00036846.2011.610750

Krueger, N. F., \& Brazeal, D. V. (1994). Entrepreneurial potential and potential entrepreneurs. Entrepreneurship Theory and Practice, 18, 91-91.

Krueger, N. F., Jr., Reilly, M. D., \& Carsrud, A. L. (2000). Competing models of entrepreneurial intentions. Journal of Business Venturing, 15(5/6), 411-432. doi:10.1016/S0883-9026(98)00033-0

Latham, G. P., \& Locke, E. A. (1991). Self-regulation through goal setting. Organizational Behavior and Human Decision Processes, 50(2), 212-247.

Mitchell, J. R., Shepherd, D. A., \& Sharfman, M. P. (2011). Erratic strategic decisions: When and why managers are inconsistent in strategic decision making. Strategic Management Journal, 32(7), 683-704. doi:10.1002/smj.905/full

Nabi, G., \& Liñán, F. (2013). Considering business start-up in recession time: The role of risk perception and economic context in shaping the entrepreneurial intent. International Journal of Entrepreneurial Behavior \& Research, 19(6), 633-655. doi:10.1108/IJEBR10-2012-0107

Nguyen, T. D. (2011). Phưong pháp nghiên cứu khoa học trong kinh doanh - Thiết kế và thưc hiện [Methods of scientific research in business - Design and implementation]. Hanoi, Vietnam: Nhà xuất bản Lao động Xã hội.

Parks-Stamm, E. J., Gollwitzer, P. M., \& Oettingen, G. (2007). Action control by implementation intentions: Effective cue detection and efficient response initiation. Social Cognition, 25(2), 248-266. doi:10.1521/soco.2007.25.2.248

Randall, D. M., \& Wolff, J. A. (1994). The time interval in the intention-behaviour relationship: Meta-analysis. British Journal of Social Psychology, 33(4), 405-418. doi:10.1111/j.20448309.1994.tb01037.x

Reynolds, P., Bosma, N., Autio, E., Hunt, S., De Bono, N., Servais, I., . . . Chin, N. (2005). Global entrepreneurship monitor: Data collection design and implementation 1998-2003. Small Business Economics, 24(3), 205-231. doi:10.1007/s11187-005-1980-1

Schlaegel, C., \& Koenig, M. (2014). Determinants of entrepreneurial intent: A meta-analytic test and integration of competing models. Entrepreneurship Theory and Practice, 38(2), 291-332. doi:10.1111/etap.12087

Schumpeter, J. A. (1934). The theory of economic development: An inquiry into profits, capital, credit, interest, and the business cycle. Cambridge, MA: Harvard University Press.

Shane, S., \& Venkataraman, S. (2000). The promise of entrepreneurship as a field of research. Academy of Management Review, 25(1), 217-226. doi:10.5465/amr.2000.2791611 
Shapero, A., \& Sokol, L. (1982). The social dimensions of entrepreneurship. Retrieved October 11 , 2017 ,

from https://www.researchgate.net/publication/228133594_The_Social_Dimensions_of_Entr epreneurship

Sheeran, P., \& Silverman, M. (2003). Evaluation of three interventions to promote workplace health and safety: Evidence for the utility of implementation intentions. Social Science \& Medicine, 56(10), 2153-2163. doi:10.1016/S0277-9536(02)00220-4

Sheeran, P., Aubrey, R., \& Kellett, S. (2007). Increasing attendance for psychotherapy: Implementation intentions and the self-regulation of attendance-related negative affect. Journal of Consulting and Clinical Psychology, 75(6), 853-863. doi:10.1037/0022006X.75.6.853

Sokol, L. (1982). The social dimension of entrepreneurship. In The encyclopedia of entrepreneurship. Englewood Cliffs, NJ: Prentice-Hall.

Souitaris, V., Zerbinati, S., \& Al-Laham, A. (2007). Do entrepreneurship programmes raise entrepreneurial intention of science and engineering students? The effect of learning, inspiration and resources. Journal of Business Venturing, 22(4), 566-591. doi:http://dx.doi.org/10.1016/j.jbusvent.2006.05.002

Stevenson, H. H., \& Jarillo, J. C. (2007). A paradigm of entrepreneurship: Entrepreneurial management. Entrepreneurship: Concepts, theory and perspective, 11, 155-170.

Tubbs, M. E., \& Ekeberg, S. E. (1991). The role of intentions in work motivation: Implications for goal-setting theory and research. Academy of Management Review, 16(1), 180-199.

van Gelderen, M., Brand, M., van Praag, M., Bodewes, W., Poutsma, E., \& van Gils, A. (2008). Explaining entrepreneurial intentions by means of the theory of planned behaviour. Career Development International, 13(6), 538-559. doi:10.1108/13620430810901688

Walker, J. K., Jeger, M., \& Kopecki, D. (2013). The role of perceived abilities, subjective norm and intentions in entrepreneurial activity. The Journal of Entrepreneurship, 22(2), 181202. doi:10.1177/0971355713490621 